\title{
Enhanced Supercontinuum Generation in Highly Nonlinear fibre by Selected Feedback Seeding
}

\author{
Yuhua $\mathrm{Li}^{1}$, Paul Barclay ${ }^{2}$, Orly Yadid-Pecht ${ }^{1}$ \\ ${ }^{1}$ Department of Electrical and Computer Engineering, University of Calgary \\ ${ }^{2}$ Department of Physics, University of Calgary \\ 2500 University Dr NW, Calgary, AB, Canada, orly.yadid.pecht@ucalgary.ca
}

\begin{abstract}
We propose a simple approach to control the supercontinuum generation, where a minute fraction of the output at specific positions was selected using a narrowband filter and then fed into a highly nonlinear fibre as the seeding light. We numerically investigated the influence of the feedback component on the supercontinuum generation. The results show the supercontinuum can be significantly enhanced with proper selected back seeding.
\end{abstract}

Keywords — Nonlinear optics, fibres, supercontinuum generation, feedback, seeding

\section{INTRODUCTION}

Supercontinuum (SC) sources have become an exciting tool for many practical and commercial applications [1]. Because the strong dependence of the SC generation process on initial conditions $[1,2]$, it has been proposed to introduce additional parameters to actively control the SC generation process and improve specific SC properties. For example, by adding an external seed light or by modulating the pump pulse envelopes [3-5]. A different approach is to utilize a feedback to influence the SC generation, where a fraction of the generated SC was used as a seed for the following pump pulse [6-8]. But until now, control of the SC generation and its properties still remains a big challenge.

In this work, we use a simple method to improve the properties of the generated SC. A minute fraction of the generated SC was extracted and then a specific part was selected by use of a narrowband filter, and finally feedback to the input end, to enable a seeded SC generation. We investigated the influence of selected feedback in SC generation in a highly nonlinear fibre (HNLF) via numerical simulation.

\section{NUMERICAL SimULATION MODEL}

We use the generalized nonlinear Schrödinger equation to model the pulse propagation inside the highly nonlinear fibre, and use a feedback loop to investigate the feedback effect on $\mathrm{SC}$ generation. The schematic diagram is presented in Fig. 1. $E_{\text {pump }}$ is the electric pump pulse envelope which was adapted to a Gaussian field profile. The approach is similar to [7]: after integrating the GNLSE with the pump pulse field profile as

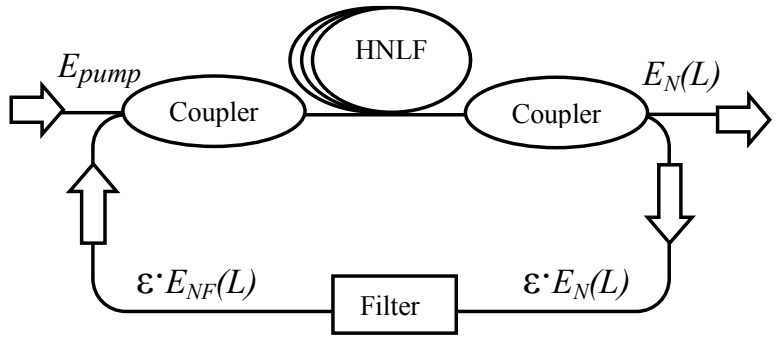

Fig. 1 Schematic diagram of the SC feedback system.

the initial condition $\left.E_{N}(\mathrm{z}=0)\right|_{n=0}=E_{0}(0)=E_{\text {pump }}(n=$ number of feedback iterations), the SC pulse envelope $E_{0}(L)=$ $\operatorname{GNLSE}\left(E_{0}(0)\right)(L=$ fiber length) at the output end of the fiber could be obtained. A small percent of the generated SC was extracted by an output splitter to the feedback loop. The specific range of the output was then selected by a narrowband filter, marked as $E_{0 F}(L)$, and finally superimposed onto the following pump pulse, by adding up the two input fields, resulting in $E_{1}(0)=\left(\varepsilon \cdot E_{0 \mathrm{~F}}(L)+E_{\text {pump }}\right)$. In order to get the next SC pulse, the GNLSE was integrated along the HNLF length again with the modified input conditions, as $E_{1}(L)=$ $\operatorname{GNLSE}\left(E_{1}(0)\right)$. This procedure was repeated for calculating the multiple selected feedback seeds.

\section{RESULTS AND DISCUSSION}

The supercontinuum was influenced by many factors, such as input pulse parameters and the nonlinear fibre parameters, and also by the feedback efficiency, feedback wavelength, and dispersion introduced by the feedback loop [6, 7]. The HNLF used for this simulation has a low dispersion slope of $\sim 0.015 \mathrm{ps} /\left(\mathrm{nm}^{2} . \mathrm{km}\right)$ and a high nonlinearity of $\sim 0.0116 /(\mathrm{W} . \mathrm{m})$, which is in favour of various nonlinear processes. We start the investigation with the pump wavelength of $1590 \mathrm{~nm}$ which is close to the zero dispersion wavelength of the fibre $(1584 \mathrm{~nm})$. So the modulation instability (MI) gain peak can be shifted far away from the pump [3]. The peak power of injected pump pulses was $20 \mathrm{~W}$. The feedback efficiency and the central wavelength of the filter were used as the variables. SuperGaussian filters A, B, C and D with bandwidth of $5 \mathrm{~nm}$ and the central wavelength of $1630 \mathrm{~nm}$ (near the pump), $1700 \mathrm{~nm}$ (in the MI gain range), $1830 \mathrm{~nm}$ (on the edge of MI gain) and $1930 \mathrm{~nm}$ (far away from MI gain) were used to select the wavelength of interest, so that provide different pumpfeedback frequency offset. 


\section{NUSOD 2013}

With these parameters, the SC spectrum can be calculated for propagation over the $10 \mathrm{~m}$ length of the HNLF, as can be seen in Fig. 2(a)-2(f).

Fig. 2(a) shows the spectrum evolution without feedback, the result indicates the peak power is not sufficient for effective spectrum broadening. Fig. 2(b)-2(f) shows the seeded spectra evolved significantly different from the unseeded one. The output spectrum enhancement obtained from the feedback is obvious. In all cases, the feedback efficiency was identified to be $\varepsilon=1 \%$ of the electric field. When using a $1630 \mathrm{~nm}$ feedback seeding, one pair of sidebands centered around the pump was observed after 1 round trip (Fig. 2(b)), which corresponds to phase-matched four-wave mixing process. Fig. 2(c) shows the spectrum evolution after 50 round trips, which is almost stable. The SC is greatly enhanced with the $20 \mathrm{~dB}$ bandwidth of about $400 \mathrm{~nm}$. The SC spectrum with a $1700 \mathrm{~nm}$ feedback seeding, as expected, was observed to be further broadened (Fig. 2(d)). It seems the spectral revolution is dominated by amplification of the separated sidebands through four wavelength mixing (FWM). After 5m, a massive soliton eject was observed. The spectra have been found not changing much when seeded in the MI range. In Figure 3(e) and (f) the seed is shifted to the edge of the MI gain at $1830 \mathrm{~nm}$ and far away from the pump at $1930 \mathrm{~nm}$, the sidebands are slowly amplified which leads to the reduction of the enhancement.
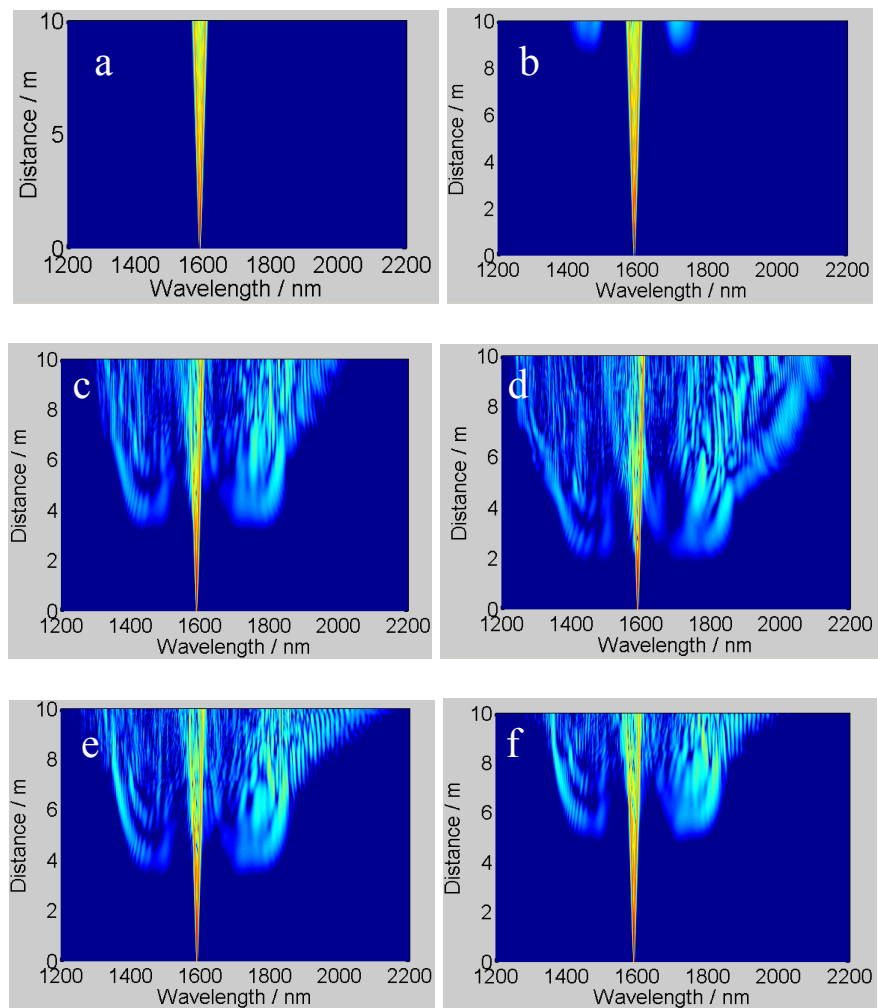

Fig. 3 Single shot simulations of pumping at $1590 \mathrm{~nm}$ with a $20 \mathrm{~W}$ pump. (a) no feedback, (b) feedback with the filter centred at $1630 \mathrm{~nm}$, first seed, (c) feedback with the filter centred at $1630 \mathrm{~nm}$, (d) feedback with the filter centred at $1700 \mathrm{~nm}$, (d) feedback with the filter centred at $1830 \mathrm{~nm}$, (d) feedback with the filter centred at $1930 \mathrm{~nm}$.

\section{CONCLUSIONS}

In conclusion, we have shown that the supercontinumm spectrum can be greatly improved by adding a selected feedback. We analyzed four typical feedback wavelengths, the results show that by selecting the feedback wavelength located in the MI gain range can considerably broaden the SC. However, the seeded SC generation is very sensitive to the initial parameters, which should be studied case by case and adjusted based on the parameters in practical experiment.

\section{REFERENCES}

[1] J. M. Dudley, G. Genty, and S. Coen, "Supercontinuum generation in photonic crystal fiber," Rev. Mod. Phys., vol. 78, pp. 1135-1184, 2006.

[2] John. M. Dudley, G. Genty, and B. J. Eggleton, "Harnessing and control of optical rogue waves in supercontinuum generation", Opt. Express vol. 16, pp. 3644-3651, 2008

[3] Simon Toft Sørensen, Casper Larsen, Uffe Møller, Peter M. Moselund, Carsten L. Thomsen, and Ole Bang, "Influence of pump power and modulation instability gain spectrum on seeded supercontinuum and rogue wave generation", J. Opt. Soc. Am. B, vol. 29, pp. 2875-2885, 2012.

[4] D. R. Solli, C. Ropers, and B. Jalali, "Active control of rogue waves for stimulated supercontinuum generation," Phys. Rev. Lett., vol. 101, 233902, 2008

[5] K. K. Y. Cheung, C. Zhang, Y. Zhou, K. K. Y. Wong, and K. K. Tsia, "Manipulating supercontinuum generation by minute continuous wave," Opt. Lett., vol. 36, pp. 160-162, 2011.

[6] P. M. Moselund, M. H. Frosz, C. L. Thomsen, and O. Bang, "Backseeding of higher order gain processes in picosecond supercontinuum generation," Opt. Express, vol. 16, pp. 11954-11968, 2008

[7] Michael Kues, Nicoletta Brauckmann, TillWalbaum, Petra Groß, and Carsten Fallnich, "Nonlinear dynamics of femtosecond supercontinuum generation with feedback," Opt. Express, vol. 17, pp 15827-15841, 2009

[8] N. Brauckmann, M. Kues, T. Walbaum, P. Groß, and C. Fallnich, "Experimental investigations on nonlinear dynamics in supercontinuum generation with feedback," Opt. Express, vol. 18, pp. 7190-7202, 2010. 